\title{
EVALUATION OF MTA AND BIODENTINE AS A PRIMARY TEETH FURCATION REPAIR
}

\author{
Manar Abdelfattah Mohammed*, Mervat Abdel Moniem Rashed**, \\ Nihal Refaat Kabel ${ }^{* * *}$ and Yomna Said Abdel Azim*
}

\begin{abstract}
Purpose: Evaluation of the sealing ability of MTA (ENDOCEM Zr) and Biodentine ${ }^{\mathrm{TM}}$ in repairing the primary molar furcation perforation by using push-out bond strength test and leakage test with dye material.

Materials and Methods: Furcation perforations were made in 60 primary molars. Molars were divided randomly on the basis of the repair material used. Thirty molars were repaired by MTA (ENDOCEM Zr) and the other thirty molars were repaired by Biodentine ${ }^{\mathrm{TM}}$. Push out bond strength test was measured and analyzed for fifteen molars of each material by using universal testing machine (Model LRX- plus, Lloyd instruments Ltd., fareham, UK). Leakage test with dye penetration was performed for fifteen molars for each material by using USB digital microscope. The images were captured and transferred to a computer equipped with the image analysis software programed. The data were statistically analyzed using Student t- test.
\end{abstract}

Results: Although MTA (ENDOCEM Zr) recorded higher push- out bond strength mean value $(4.759 \pm 1.84 \mathrm{MPa})$ than Biodentine $\mathrm{T}^{\mathrm{TM}}(3.449 \pm 1.30 \mathrm{MPa})$ but it was statistically non- significant $(\mathrm{t}=$ $0.89, \mathrm{P}=0.4096 \geq 0.05)$. while Biodentine ${ }^{\mathrm{TM}}$ recorded, slight higher leakage mean value $(0.951433 \pm$ $0.38 \mathrm{~mm})$ than MTA (ENDOCEM Zr) $(0.946229 \pm 0.29 \mathrm{~mm})$ it was also statistically non- significant $(\mathrm{t}=0.02, \mathrm{P}=0.9835 \leq 0.05)$.

Conclusion: MTA (ENDOCEM Zr) and Biodentine ${ }^{\mathrm{TM}}$ showed comparable seal for furcation perforation of primary molars.

\section{INTRODUCTION}

The main objective of pulp therapy in primary teeth is to maintain the integrity of oral structures, guide permanent teeth to erupt properly and finally ensure normal well-being of the child ${ }^{[1]}$. Pulp treatment in primary teeth is considered difficult due to many reasons. These include the complexity of pulp treatments and behavior challenges in

* Department of Pediatric and Community Dentisty, Faculty of Oral and Dental medicine, October 6 University, Giza, Egypt

** Department of Pediatric Dentisty and Dental Public Health, Faculty of Oral and Dental Medicine, Cairo University, Giza, Egypt

*** Department of Pediatric Dentisty and Dental Public Health, Faculty of Oral and Dental Medicine, Misr University for Science and Technology, Giza, Egypt 
children $^{[2,3]}$, so accidental furcal perforation can be induced. Furcal perforation is a communication between root canals and periodontal ligaments through the pulp chamber ${ }^{[1,2]}$. It is considered as an undesirable incident which affects the prognosis of the treatment. It can be occurring due to different reasons, including rampant caries, resorption and the misdirection of the bur in preparing the access cavity of the pulp chamber. Perforation has been reported to occur in $2-12 \%$ of cases ${ }^{[4]}$.

Any delay in repairing results in the bacterial contamination of the perforation site which consequently leads to the gingival down-growth of the epithelium into the perforation area, inflammation, bone resorption, necrosis and eventual loss of the tooth ${ }^{[5,6]}$.Primary molar teeth are involved in mastication, speaking and esthetics, therefore, keeping them till eruption of permanent teeth eruption is unavoidable ${ }^{[1,7]}$. The repairing of furcal perforation can be achieved by using non-surgical or surgical approach and employing different materials such as: amalgam, IRM, Guttapercha, light- cured GI cement, resin composite, MTA, calcium hydroxide and Biodentine as reported in many studies in the literature ${ }^{[2,5,8-10]}$

Ideally, the material used for sealing of root perforations should be atoxic, non-resorbable, radiopaque and bacteriostatic and should have good seal ability ${ }^{[4,5]}$. In addition, the material should present an osteogenic inductive capacity and biocompatibility ${ }^{[5]}$. Taking into account these characteristics and prerequisites, the use and/or behavior of different materials for sealing of root perforations has been investigated ${ }^{[6-8]}$.

Mineral trioxide aggregate (MTA) was developed in the 1990s by Torabinejad and his coworkers at Loma Linda University (Loma Linda, CA); MTA is a bioactive silicate cement that is currently used for pulp therapy. MTA has been implemented successfully in the repair of lateral root and furcal perforations, apex genesis and as a vital pulp capping agent. Moreover, it was utilized as an apical plug in one visit apexification and as a rootend filling material ${ }^{[9,10]}$. MTA has many properties inducing: hard tissue formation adjacent to pulp, low toxicity, antibacterial effect and inducing cementogenesis ${ }^{[11-14]}$.

Some studies have compared the clinical and histologic outcomes of direct pulp capping with MTA (ProRoot MTA; Dentsply, Tulsa, OK) with those of calcium hydroxide ${ }^{[1-4]}$ Most of these trials have reported that the clinical and histologic responses achieved through the use of MTA are similar or superior to those achieved with calcium hydroxide ${ }^{[11]}$. MTA is hygroscopic, and its ability to set is not affected by the presence of blood or serum fluid. ${ }^{[11-15]}$ The close physiochemical seal formed between dentin and MTA provides an insoluble barrier against microleakage. ${ }^{[16,17]}$ These properties of MTA may contribute to the success of direct pulp capping and the decreases in pulpal irritation, dystrophic calcification, and potential degenerative changes in the pulp that are associated with the use of calcium hydroxide. ${ }^{[13]}$

MTA is slightly more radiopaque than dentin, has low solubility and its compressive strength twentyone days after setting is $70 \mathrm{MPa}$, which allows restorative materials to be condensed over a MTA base without affecting its structure ${ }^{[7]}$. However, to date, only a few studies have investigated the biocompatibility and physical properties of Endocem in vitro. ${ }^{[12-14]}$

MTA has resulted in successful outcomes in furcation repair. Despite many good properties ${ }^{[9]}$, MTA has long setting time and difficult handling ${ }^{[5,9-12]}$. These are important considerations for clinical application in pediatric patients. In two separate studies, the investigators found no significant difference between MTA and CEM cement as perforation repair materials in primary molars ${ }^{[2]}$. There are scarce papers that have evaluated the applications of Biodentine as a 
posterior restorative material, the capping agent in vital pulp therapies and root end filling ${ }^{[1,16,17]}$

Endocem (Maruchi, Wonju, Korea) is an MTA-derived pozzolan cement that was recently introduced for pulp therapy and was approved in 2012 by the US Food and Drug Administration. The chemical composition of Endocem is similar to that of MTA, and Endocem has some advantages over MTA that include rapid setting and favorable manipulation properties. ${ }^{[18-21]}$

Endocem $\mathrm{Zr}$ is a zirconium oxide-enriched calcium silicate-based cement and its high $\mathrm{Zr}$ content. Zirconium oxide has been employed as a radiopacifier of calcium silicate-based endodontic materials as a substitute of bismuth oxide, because bismuth oxide retards the setting reaction of MTA. ${ }^{[19]}$ Zirconium oxide nanoparticles have also been reported to accelerate the degree of hydration of Portland cements. Thus, the replacement of bismuth oxide with zirconium oxide may contribute to the fast-setting property of Endocem $\mathrm{Zr}$. ${ }^{[5]}$

Biodentine is a calcium silicate-based bioactive material. It is a powder liquid system, powder composed of Tri-calcium silicate, Di-calcium silicate, Calcium carbonate and oxide, Iron oxide, Zirconium oxide. Liquid consist of Calcium chloride, Hydro soluble polymer. ${ }^{[13,22]}$ It is easy to handle owing to its ease of manipulation and a short setting time approximately 12 minutes, has high alkaline $\mathrm{pH}$ and is a biocompatible material makes it a favorable material for perforation repair ${ }^{[23]}$ Biodentine bonds chemo-mechanically with the tooth and composite. ${ }^{[13]}$ Biodentine is dentine substitute containing tricalcium silicate with good handling and mechanical properties. It has also good sealing ability and short setting time $[13,15,22,23]$

Push-out test is a test to measure the interfacial shear strength developed between different surfaces. It provides information about the adhesive property of the material tested ${ }^{[1]}$ and helps to understand the resistance of the tested material to dislodgement, that is how well the material can bind to the tooth structure. The greater the push-out strength value, the greater is the adhesion between the tested material and the tooth surface. In endodontics, the push-out bond strength is done for root end filling, perforation repair, obturation, and root canal sealer materials, to study their resistance to dislodgement ${ }^{[15,23,24]}$

This invitro study was done for evaluating the ability of MTA (ENDOCEM Zr) and Biodentine ${ }^{\mathrm{TM}}$ to seal furcation perforation of primary molars using push-out bond strength test and leakage test with dye material.

\section{Purpose:}

the aim of this study was to evaluate the sealing ability of MTA (ENDOCEM Zr) and Biodentine ${ }^{\mathrm{TM}}$ in repairing the primary molar furcation perforation by using push-out bond strength test and leakage test with dye material.

\section{MATERIALS AND METHODS}

Endocem Zr (Lot No. ZE8805110819) and Biodentine (Lot No. B02282; Septodent, USA) were used. These materials were mixed with distilled water according to the manufacturer's instruction.

Specimens' preparation and grouping:

1- In this invitro study, 60 freshly extracted primary mandibular second molars were used. The sample size was calculated based on previous similar studies ${ }^{[1-3]}$ using Minitab statistical software. ${ }^{[1]}$ The inclusion criteria were as follows: normal furcation (with completely distinct roots), minimal caries (at least $4 \mathrm{~mm}$ caries free surfaces above the CEJ), and no previous pulp treatment. Teeth with cracks were excluded after microscopic inspection. After cleaning, washing and disinfecting, the samples were kept in 5\% sodium hypochlorite for 30 minutes) 
2- In each tooth, a standardized access cavity was prepared using a \#2 diamond bur (D\&Z Co., Wies Baden, Germany) mounted in a highspeed water cooled handpiece and the root canal orifices were located. Molars were amputated $3 \mathrm{~mm}$ below the furcation area using a tapered diamond stone. (Figure1).

3- Sticky wax was placed over the orifices of each canal. It was then coated with two successive layers of varnish in an attempt to increase the marginal seal (Figure 2).

4-A standardized artificial perforation of $1 \mathrm{~mm}$ in diameter was created from the external surface of the tooth with a $\# 2$ round carbide bur mounted on a high-speed hand piece with air water coolant the chamber and perforation were flushed with water and dried. The size of perforation was the

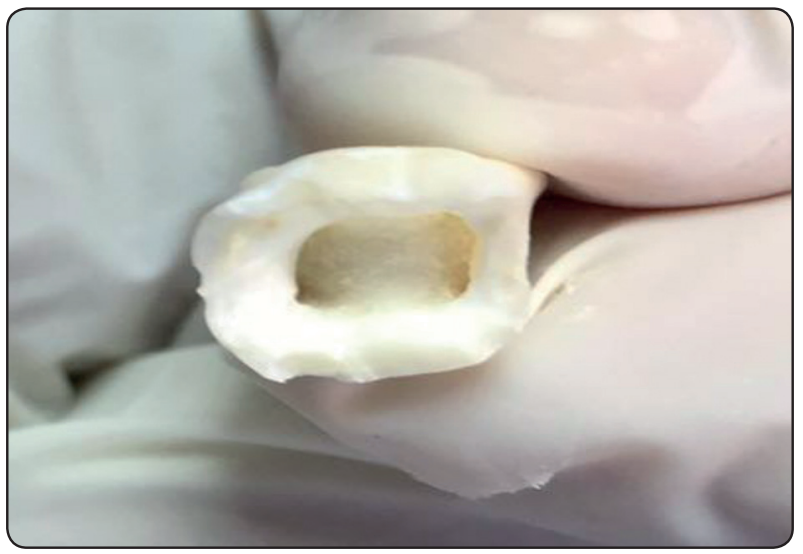

Fig. (1) A standardized access cavity preparation. same as the bur size (1 $\mathrm{mm}$ in diameter) in all samples. The bur was replaced with a new one after making every six perforations.

5- The samples were randomly assigned into two groups (two experimental groups). Group I $(n=30)$, perforations were sealed with MTA (ENDOCEM Zr (Maruchi, Wonju, Korea)) and group II $(n=30)$ perforations were sealed with Biodentine (Septodont, Saint-Maur-des-Fosses, France), respectively. After irrigation of samples with $10 \mathrm{~mL}$ normal saline, repairing materials were mixed according to the manufactures' instructions and placed by a carrier gun on the perforation site. Biomaterials were packed with moist cotton pellets while the samples were positioned in wet soft sponges. Condensing upon the sponge simulated the clinical condition in the oral cavity. At the end, all samples were

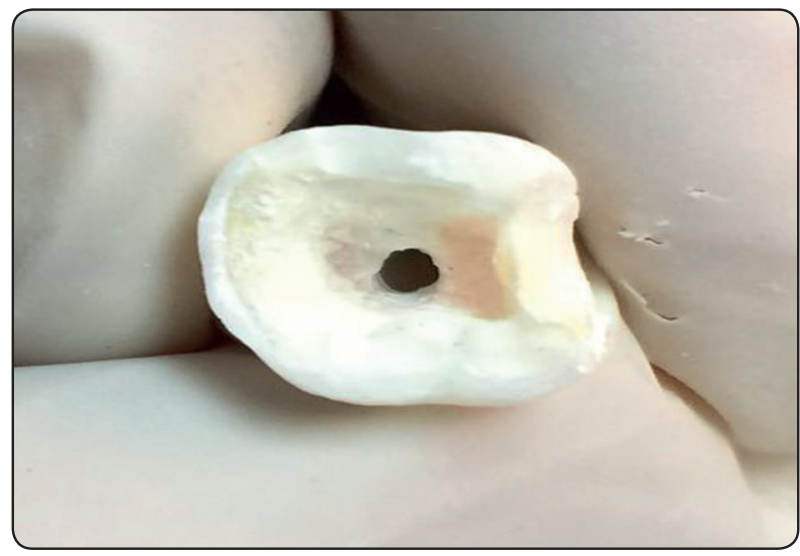

Fig. (2) Sticky wax placing over the orifices of each canal.
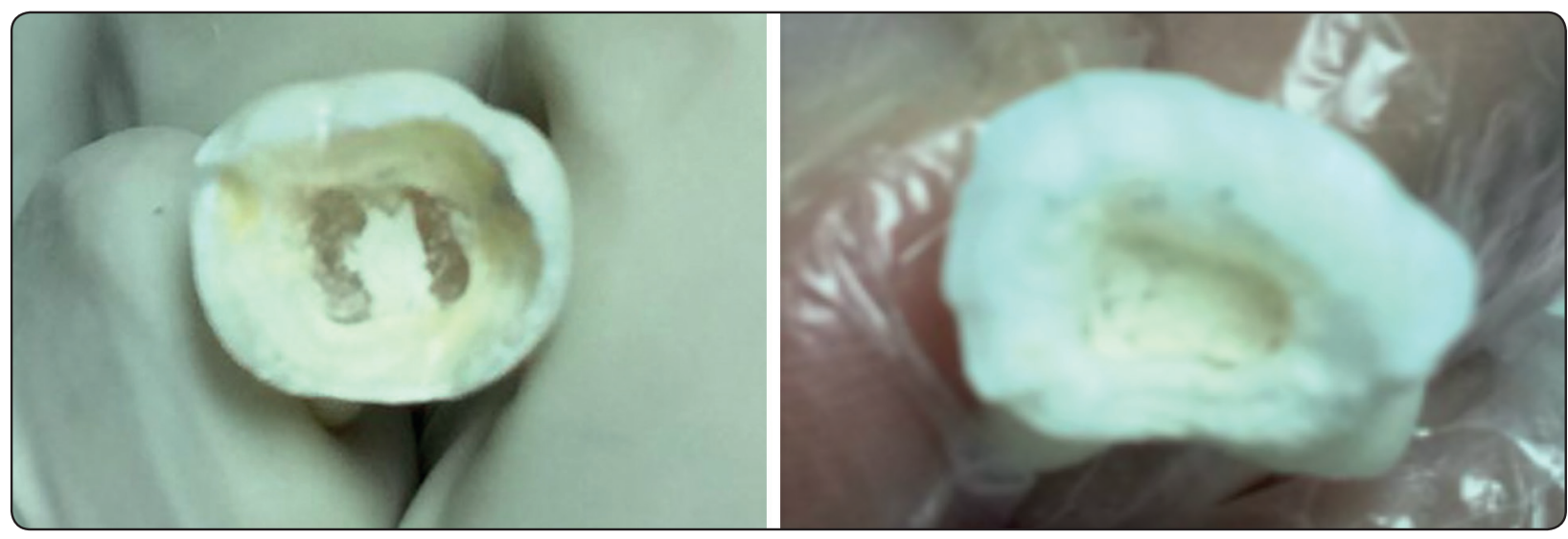

Fig. (3) Tooth after perforation repairing by tested materials (MTA ENDOCEM Zr\& BiodentinTM) 
placed in an incubator at $37^{\circ} \mathrm{C}$ and $100 \%$ humidity for $24 \mathrm{~h}$ to allow the biomaterials being fully set. ${ }^{[1]}$

\section{A) Push out test strength:}

1- Push out bond strength test was measured and analyzed for fifteen molars of each material by using universal testing machine (Model LRXplus, Lloyd instruments Ltd., fareham, UK).

2- The samples were placed on a metal slab with a central hole to allow the free motion of the plunger.

3- The compressive load was applied by exerting a download pressure on the surface of the test material in each sample with the probe moving at a constant speed of $1 \mathrm{~mm} / \mathrm{min}$. The plunger had a clearance of approximately $0.2 \mathrm{~mm}$ from the margin of the dentinal wall to ensure contact only with the test materials.

4- The maximum force which applied to materials at the time of dislodgement was recorded in newtons.

5- The push-out bond strength in megapascal (MPa) was calculated by dividing this force by the surface area of tested material. ${ }^{[23]}$

\section{B) Leakage test:}

1- Leakage test with dye penetration was performed for fifteen molars for each material by using USB digital microscope.

2- The teeth were covered with two layers of nail polish (except $1 \mathrm{~mm}$ around the restoration bottom surface - furcal-wise) then vertically immersed in a solution of $2 \%$ methylene blue dye (SD Fine-Chem limited, Mumbai, India) for 24 hours at $37 \mathrm{C}^{\circ}$. Subsequently, samples were taken out of the dye solution, washed with water, and the samples were mounted onto special holding device for sectioning.

3- Samples were sectioned with a low speed diamond saw (Top Dent, Edenta Golden, Swiss) under water spray. The specimens were rinsed in running water and then dried with tissue paper. The dye penetration along the perforation wall was assessed with USB Digital microscope (Scope Capture Digital Microscope, Guangdong, China) at $35 \times$ magnification in which the image was captured and transferred to a computer equipped with the image analysis software program (Image J 1.43U, National Institute of Health, USA). Within the Image J software, all limits, sizes, frames and measured parameters are expressed in pixels. Therefore, system calibration was done to convert the pixels into absolute real world units. Calibration was made by comparing an object of known size (a ruler in this study) with a scale generated by the Image J software. Then, the images of traced dye path were overlaid in order to calculate dye penetration depth. The total dye penetration depth along the restoration-tooth interface were measured in $(\mathrm{mm})$.

\section{Statistical analysis}

Data analysis was performed in several steps. Initially, descriptive statistics for each group results. Student t-test was performed to detect significance between groups Statistical analysis was performed using Graph-Pad Prism version 4.00 for Windows, GraphPad Software, San Diego California USA. $\mathrm{P}$ values $<0.05$ are considered to be statistically significant in all tests.

\section{RESULTS}

\section{A) Push out bond strength test results}

Push out bond strength test results $(\mathrm{Mean} \pm \mathrm{SD})$ for both groups measured in mega Pascal (MPa) are summarized in (Table 1) and graphically drawn in (Figure 4).

It was found that MTA(ENDOCEM Zr) recorded

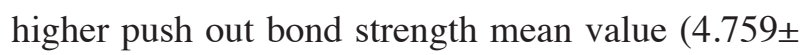
1.84MPa) than Biodentine $\mathrm{TM}^{\mathrm{TM}}(3.449 \pm 1.30 \mathrm{MPa})$

The difference between both sealers groups was statistically non-significant as indicated by student $\mathrm{t}$-test $(\mathrm{t}=0.89, \mathrm{p}=0.4096>0.05$ 


\section{B) Leakage test results:}

Leakage test results (Mean $\pm \mathrm{SD}$ ) for both groups measured with dye penetration depth (mm) summarized in (Table 2) and graphically drawn in (Figure 5)
It was found that Biodentine ${ }^{\mathrm{TM}}$ recorded higher leakage mean value $(0.951433 \pm 0.38 \mathrm{~mm})$ than MTA (ENDOCEM Zr) $(0.946229 \pm 0.29 \mathrm{~mm})$.

The difference between both group was statistically non-significant as indicated by student t-test $(\mathrm{t}=0.02, \mathrm{p}=0.9835<0.05)$

TABLE (1) Comparison of push out result (Mean values \pm SDs) between both groups.

\begin{tabular}{|c|c|c|c|c|c|}
\hline \multicolumn{2}{|c|}{ Variable } & Mean & $S D$ & Minimum & Maximum \\
\hline \multirow{2}{*}{ Push out bond } & MTA (ENDOCEM Zr) & 4.759 & 1.84 & 1.09 & 6.695 \\
\hline & Biodentine $^{\mathrm{TM}}$ & 3.449 & 1.30 & 2.00 & 5.158 \\
\hline \multirow{2}{*}{$t$-test } & t-value & \multicolumn{4}{|c|}{0.89} \\
\hline & P value & \multicolumn{4}{|c|}{$0.4096 \mathrm{~ns}$} \\
\hline
\end{tabular}

*; significant $(p<0.05)$

ns; non-significant $(p>0.05)$

TABLE (2) Comparison of leakage results (Mean values \pm SDs) between both groups.

\begin{tabular}{|l|l|c|c|c|c|}
\hline \multicolumn{2}{|c|}{ Variable } & Mean & SD & Minimum & Maximum \\
\hline \multirow{2}{*}{ Leakage } & MTA (ENDOCEM Zr) & 0.946229 & 0.29 & 0.5659 & 1.5962 \\
\cline { 2 - 6 } & Biodentine $^{\mathrm{TM}}$ & 0.951433 & 0.48 & 0.3904 & 1.6767 \\
\hline \multirow{2}{*}{$t$-test } & \multirow{2}{*}{$\boldsymbol{t}$ - value } & \multicolumn{3}{|c|}{0.02} \\
\cline { 2 - 6 } & P value & \multicolumn{2}{|c|}{$0.9835 \mathrm{~ns}$} \\
\hline
\end{tabular}

*; significant $(p<0.05) \quad$ ns; non-significant $(p>0.05)$

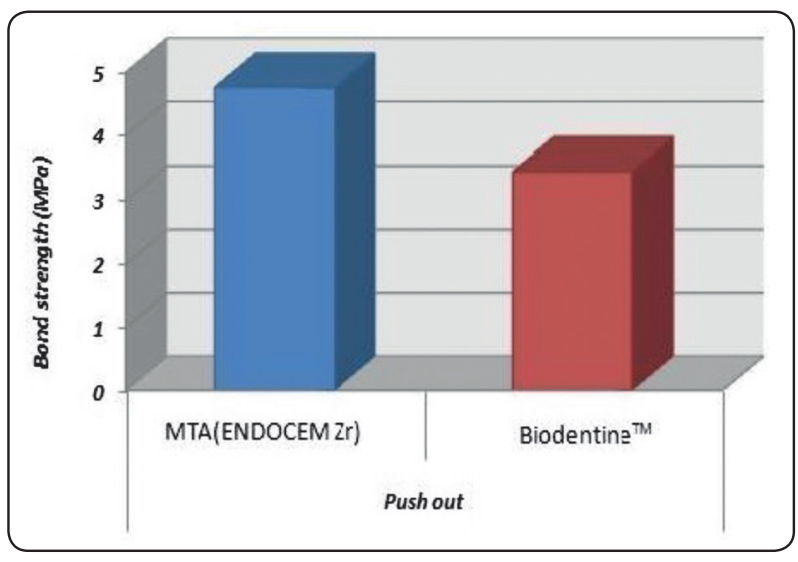

Fig. (4) Shows a column chart of push out mean values between both groups.

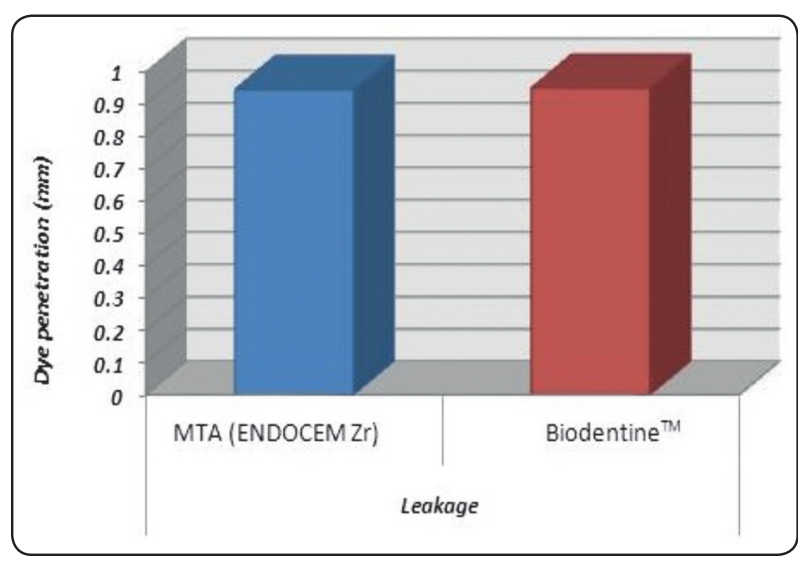

Fig. (5): Shows a column chart of leakage mean values between both groups. 


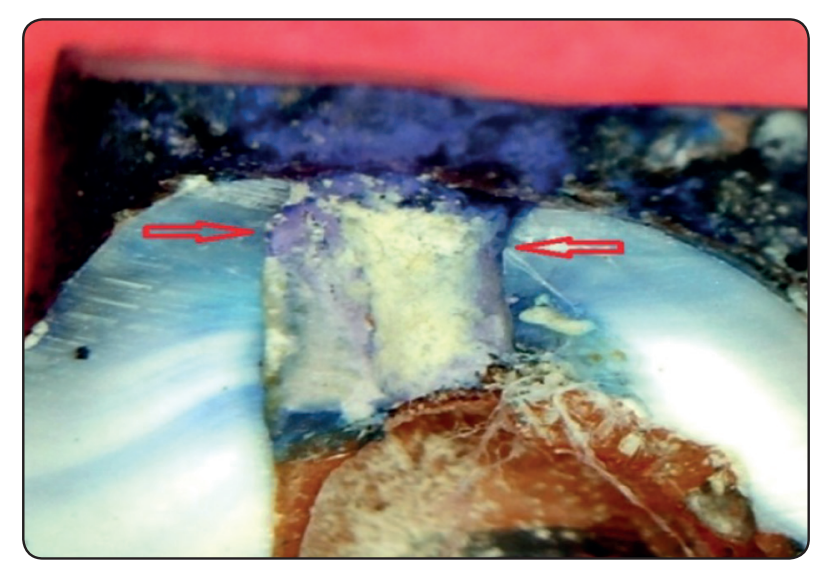

Fig. (6) Shows representative microscopic image for MTA (ENDOCEM Zr) dye penetration depth (x35).

\section{DISCUSSION}

The focus point of the current study (comparison of MTA (Endcem Zr) and Biodentine based on the sealing ability as perforation repair materials) have not been explored in earlier studies. based on statistical tests throughout the dye penetration and push out experiments, the two perforation repair materials had no significant different.

Several studies have reported the ability of MTA to prevent leakage in a variety of applications ${ }^{[1-4]}$. Moreover, they reported its superiority compared to other dental materials. For this reason, we included MTA (Endocem Zr) as a standard perforation repair material for better comparison.

Different methods are used to evaluate the microleakage of restoration, including silver nitrate, air pressure, bacteria, radioactive isotopes, organic dyes, calcium hydroxide technique and scanning electron microscope (SEM). ${ }^{[20]}$

Dye penetration is considered as a successful method because of its easy penetration into the flaws and crevices of the test object. Some of the organic dyes used include basic fuchsin, methylene blue, eosin, aniline blue, crystal violet and erythrosin B [18]. In this study, methylene blue solution was used because it can penetrate better than other solutions

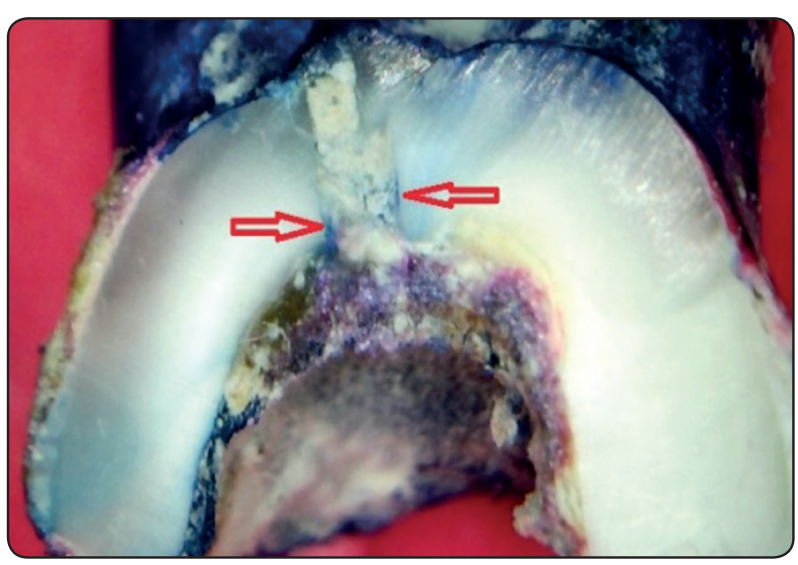

Fig. (7) Shows representative microscopic image for Biodentine ${ }^{\mathrm{TM}}$ dye penetration depth (x35).

due to its size that is smaller than the smallest bacteria. On the other hand, the dye permits an easy visualization of the sample cavities and excellent contrast with the surrounding environment and it is inexpensive ${ }^{[17]}$.

In another study, regarding the sealing ability of different materials, significantly higher microleakage scores of MTA were reported compared to CEM cement. ${ }^{[18]}$

Biodentine $^{\mathrm{TM}}$ is very similar to MTA in basic composition. The manufacturers claim that the addition of setting accelerators and softeners, a new pre-dosed capsule formulation for use in a mixing device predominantly improves the physical properties of the material, making it more userfriendly. Biodentine ${ }^{\mathrm{TM}}$ does not require two-step obturation as the setting is faster and thus is lower risk of bacterial contamination making it superior to MTA. ${ }^{[24]}$

The current study showed that Biodentine ${ }^{\mathrm{TM}}$ recorded mean value $(0.951433 \pm 0.38 \mathrm{~mm})$, while MTA (ENDOCEM Zr) $(0.946229 \pm 0.29 \mathrm{~mm})$, this result showed insignificant difference which disagree with other study that presented Biodentine ${ }^{\mathrm{TM}}(0.149$ $\pm 0.097)$ has lesser microleakage values compared to MTA $(0.583 \pm 0.24)$, these different findings may be attributed to different techniques and methodology 
used to evaluate microleakage and the type of tooth material (primary versus permanent teeth). ${ }^{[22]}$

In one study, it was showed that when Biodentine was used as root-end filling material, significantly better marginal adaptation was observed compared to MTA [18]. However, according to Soundappan et al. ${ }^{[24]}$, Biodentine could not compete with MTA as root end filling material. Considering the biocompatible entity of Biodentine and its ability to induce odontoblast differentiation the bacterial leakage resistance of this calcium-silicate cement after repair of perforation must be assessed.

The finding of this study confirms the previous investigation, which evaluated the sealing ability of MTA Plus ${ }^{\mathrm{TM}}$ and Biodentine ${ }^{\mathrm{TM}}$.by using dye penetration model in primary molar. It was concluded that the two testing biomaterials demonstrate similar capacities as furcation perforation materials. ${ }^{[3]}$

Despite the promising results regarding the sealing ability of Endocem $\mathrm{Zr}$ and Biodentine, it should be kept in mind that in vitro studies, due to many inherent drawbacks cannot simulate oral condition completely. On the other hand, because of no expression of full clinical characteristic of the repairing material under in vitro conditions, the long-term prognosis of perforation sealed teeth is unknown. So, future clinical studies on accidentally perforated primary molars are recommended to evaluate the long-term prognosis.

The perforations of furcation of primary molars may interfere with the prognosis of treatment. [9] Biocompatible material should be used immediately for sealing the communication between root canal system and external root surfaces ${ }^{[9]}$. The present study evaluated the sealing of MTA (ENDOCEM $\mathrm{Zr}$ ) and Biodentine ${ }^{\mathrm{TM}}$ as furcation repair materials in primary molars.

The bond strength of endodontic materials which used for perforation repair is an important factor in clinical practice. One of the requirements of such material is to persist in place under dislodging forces, such as mechanical stress resulting from tooth furcation or operative procedures. Different methods have been used to demonstrate the adhesive properties of dental materials to the surrounding dentin $^{[22,21]}$. This include tensile, shear and push-out bonding strength test. The push-out bond strength which was used in this study has been shown to be efficient, practical and reliable. ${ }^{[20]}$ The published research on the bond strength for biodentine for primary teeth is very limited, this study done to compare the push-out strength between ENDOCEM $\mathrm{Zr}$ and Biodentin ${ }^{\mathrm{TM}}$. For standardization, the two materials were tested after the same period of time elapsed since mixing.

Although MTA (ENDOCEM Zr) recorded higher push out bond strength mean value (4.759 $\pm 1.84 \mathrm{MPa})$ than Biodentine $\mathrm{T}^{\mathrm{TM}}(3.449 \pm 1.30 \mathrm{MPa})$, The difference between both sealer groups was statistically non-significant. This result showed disagreement with Guneser et al who concluded that the force needed for the displacement of Biodentine $^{\mathrm{TM}} 7.12 \pm 3.10 \mathrm{MPa}$ from root dentin was significantly higher than MTA $3.03 \pm 1.28 \mathrm{MPa}$ [23]. Comparable results between ENDOCEM Zr and Biodentin ${ }^{\mathrm{TM}}$ may be due to using primary teeth instead of permanent which have different structure. New generation of MTA with improved mechanical characteristics leading to comparable result to Biodentin. ${ }^{\mathrm{TM}}$ The good properties of Biodentine as reported in our findings makes the addition of this cement to the list of primary teeth perforation repair materials is crucial.

\section{CONCLUSION}

MTA(ENDOCEM Zr) and Biodentine ${ }^{\mathrm{TM}}$ showed comparable seal for furcation perforation of primary molars. 


\section{REFERENCES}

1. Ramazania N, and Sadeghib P.Bacterial Leakage of Mineral Trioxide Aggregate, Calcium-Enriched Mixture and Biodentine as Furcation Perforation Repair Materials in Primary Molars, Iran Endod J. 2016 Summer; 11(3): 214-218.

2. Haghgoo, R, Niyakan A M, Nazari Moghaddam B M, Asgary C S, and Mostafaloo N, An In vitro Comparison of Furcal Perforation Repaired with Pro-root MTA and New Endodontic Cement in Primary Molar Teeth- A Microleakage StudyJ Dent (Shiraz). 2014 Mar; 15(1): 28-32.

3. Farhin A. Katge, Pooja Ravindra Shivasharan, and Devendra Patil Sealing ability of mineral trioxide aggregate Plus $^{\mathrm{TM}}$ and Biodentine ${ }^{\mathrm{TM}}$ for repair of furcal perforation in primary molars: An in vitro study Contemp Clin Dent. 2016 Oct-Dec; 7(4): 487-492.

4. Samuel A, Asokan S, P. R. Geetha, and Thomas S. Evaluation of sealing ability of Biodentine ${ }^{\mathrm{TM}}$ and mineral trioxide aggregate in primary molars using scanning electron microscope: A randomized controlled in vitro trialContemp Clin Dent. 2016 Jul-Sep; 7(3): 322-325

5. Han L., Kodama S, Okii T. Evaluation of calcium-releasing and apatite-forming abilities of fast-setting calcium silicate-based endodontic materials Inter endod J ,Volume 48, Issue 2 Pages 124-130 February 2015.

6. Silveira CMM, Sánchez-Ayala A, Lagravère MO, Pilatti GL, Comes OMM. Repair of furcal perforation with mineral trioxide aggregate: long-term follow-up of 2 cases. J Can Dent Assoc. 2008;74(8):729.

7- Bidar M, Dysfani R, Gharehchaghi M, Tabatabaii R. Laboratory evaluation of microleakage of one coat bond, Root MTA, ProRoot MTA in restoring of furca perforation. J Dent Isfahan Uni Med Sci. 2007;3:76-81.

8. De Deus G, Petruccelli V, Gurgel Filho E, CoutinhoFilho T. MTA versus Portland cement as repair material for furcal perforations: a laboratory study using a polymicrobial leakage model. Int Endod J. 2006;39(4):293-8.

9. Nakata TT, Bae KS, Baumgartner JC. Perforations repair comparing MTA and amalgam using anaerobic bacterial leakage model. J Endod. 1998;24:184-186.

10. Sluyk SR, Moon PC, Hartwell GR. Evaluation of setting properties and retention characteristics of MTA when used as furcation perforation repair material. J Endod. 1998;24:768-771.
11. Juárez Broon N, Bramante CM, Assis GF, Bortoluzzi EA, Bernardineli N, Moraes IG, et al. Healing of root perforations treated with Mineral Trioxide Aggregate (MTA) and Portland cement. J Appl Oral Sci. 2006; 14:305311 .

12. Parirokh M, Torabinejad M. Mineral trioxide aggregate: a comprehensive literature review - Part III: clinical applications, drawbacks, and mechanism of action. J Endod. 2010;36:400-413.

13- Sinkar RC, Patil SS, Jogad NP, Gade VJ. Comparison of sealing ability of ProRoot MTA, RetroMTA, and Biodentine as furcation repair materials: An ulteraviolt spectrophotometric analysis. J Conserv Dent 2015; 18:445-448.

14. Bisws M, Mazumdar D, Neyogi A. Non surgical perforation repair by mineral trioxide aggregate under dental operating microscope. J Conserv Dent 2011; 14:83-5.

15. Aggarwal V, Singla M, Miglani S, Kohli S. Comparative evaluation of Push- out bond strength of ProRoot MTA, Biodentine, and MTA Plus in furcation perforation repair . J Conserv Dent 2013; 16:462-5.

16. Jeevani E, JayaprakashT, Bolla N, Vemuri S, Sunil CH, and Kalluru R Evaluation of sealing ability of MMMTA, Endosequence, and biodentine as furcation repair materials: UV spectrophotometric analysis J Conserv Dent. 2014 Jul-Aug; 17(4): 340-343.

17. Dimitrios V, Tsatsas Helen A, Meliou, Nikolaos P, Kerezoudis. Sealing effectiveness of materials used in furcation perforation in vitro. Int Dent J. 2005 Vol 55, Iss 3June 133-141.

18. Sahebi s, Moazami F, Sadat Shojaee N and Layeghneghad MK. Comparison of MTA and CEM Cement Microleakage in Repairing Furcal Perforation, an In Vitro Study. J Dent (Shiraz). 2013 Mar; 14(1): 31-36.

19. Choi Y, Park SJ, Lee SH, et al. Biological effects and washout resistance of a newly developed fast-setting pozzolan cement. J Endod 2013;39:467-72. 23. Guneser MB, Akbulut MB, Eldeniz AU. Effect of Various Endodontic Irrigants on the Push-out Bond Strength of Biodentine and Conventional Root Perforation Repair MaterialsJOE. 2013 ;39: 380-84.

20. Jang JH, Kang M, Ahn S, et al. Tooth discoloration after the use of new pozzolan cement (Endocem) and mineral trioxide aggregate and the effects of internal bleaching. $\mathrm{J}$ Endod 2013;39:1598-602. 
21. Song M, Yoon TS, Kim SY, Kim E. Cytotoxicity of newly developed pozzolan cement and other root-end filling materials on human periodontal ligament cell. Restor Dent Endod 2014;39:39-44.

22. Kokate SR, Pawar AM. An in vitro comparative stereomicroscopic evaluation of marginal seal between MTA, glass ionomer cement and biodentine as root end filling materials using $1 \%$ methylene blue as tracer. Endodontology. 2012;24:36-42

23. Kaup M, Schäfer E, Dammaschke T. An in vitro study of different material properties of Biodentine compared to
ProRoot MTA. Head Face Med. 2015;11:16.

24. Soundappan S, Sundaramurthy JL, Raghu S, Natanasabapathy V. Biodentine versus mineral trioxide aggregate versus intermediate restorative material for retrograde root end filling: An in vitro study. J Dent (Tehran) 2014;11:143-9.

25. Hashem AA, Wanees Amin SA. The effect of acidity on dislodgment resistance of mineral trioxide aggregate and bioaggregate in furcation perforations: An in vitro comparative study. J Endod. 2012;38:245-9. 